\title{
POLAR INVARIANTS OF PLANE CURVES AND THE NEWTON POLYGON
}

\author{
Andrzej Lenarcik and Arkadiusz PŁoski*
}

\begin{abstract}
We prove a factorization theorem for the polars of plane singularities with respect to the Newton diagram and calculate the polar quotients of nondegenerated singularities.
\end{abstract}

\section{Introduction}

Let $f=f(X, Y) \in C\{X, Y\}$ be a convergent power series in two variables $X$, $Y$. In all this note we assume that $f$ has an isolated singularity at $(0,0) \in$ $\boldsymbol{C}^{2}$. The polar of $f$ with respect to the direction $(a: b) \in \boldsymbol{P}^{1}(\boldsymbol{C})$ of the line $t=$ $b X-a Y$ is the power series $\partial f=\partial(t, f) / \partial(X, Y)=a(\partial f / \partial X)+b(\partial f / \partial Y)$. If the line $b X-a Y=0$ is not tangent to the curve $f=0$ then the polar quotients

$$
\frac{(f, h)_{0}}{\operatorname{ord} h}, \quad h \text { runs over irreducible divisors of } \partial f
$$

(where $(f, h)_{0}$ is $\boldsymbol{C}$ codimension of the ideal generated by $f, h$ in $C\{X, Y\}$ and ord $h$ is the order of $h$ ) are topological invariants of the singularity $f=0$ (see [LMW2]).

According to Teissier [Tel] the Łojasiewicz exponent $\mathscr{L}_{0}(f)$ defined to be the smallest $\theta>0$ such that

$$
|\operatorname{grad} f(x, y)| \geq C \max (|x|,|y|)^{\theta} \text { near }(0,0) \in C^{2},
$$

is given by the formula:

$$
\mathscr{L}_{0}(f)=(\text { the greatest polar quotient of } f=0)-1 \text {. }
$$

Merle in $[\mathbf{M}]$ proved a theorem on a factorization of the generic polar of an algebroid irreducible curve and calculated its polar quotients in terms of the characteristic. His result was generalized by different authors (see $[\mathrm{Ab} . \mathrm{A}],[\mathrm{C}]$, $[\mathrm{D}],[\mathrm{G}])$. The aim of this note is to calculate the polar quotients of a curve $f=$

1991 Mathematics Subject Classification: 32 S55.

Key words and phrases: singularity, polar quotients, Newton diagram.

* Supported in part by the KBN grant No 2 P03 A 00115.

Received June 1, 1999. 
0 nondegenerate in the sense of Kouchnirenko [Kou] in terms of the Newton polygon of $f$. Our main result gives a factorization of the generic polar curve and is parallel to Merle's theorem (see [M], Théorème 3.1). As an application we give a proof of the formula for the Lojasiewicz exponent obtained recently by the first author [Len]. The formula is an improvement of an earlier result due to Lichtin [Li].

\section{Main result}

Let $f \in C\{X, Y\}$ be a nonzero power series without constant term. Write $f=\sum c_{\alpha \beta} X^{\alpha} Y^{\beta}$ and $\operatorname{supp} f=\left\{(\alpha, \beta) \in N^{2}: c_{\alpha \beta} \neq 0\right\}$. The Newton polygon $\mathscr{N}(f)=\mathscr{N}_{f}$ is the set of the compact faces of the boundary of the convex hull $\Delta(f)$ of $\operatorname{supp} f+N^{2}$. For any segment $S \in \mathscr{N}_{f}$ we let in $(f, S)=$ the sum of all monomials $c_{\alpha \beta} X^{\alpha} Y^{\beta}$ such that $(\alpha, \beta) \in S$. According to Kouchnirenko [Kou], the series $f$ is nondegenerate on $S \in \mathcal{N}_{f}$ if the polynomial in $(f, S)$ has no critical points in the set $C^{*} \times C^{*}$, where $C^{*}=C \backslash\{0\}$. The series in nondegenerate if it is nondegenerate on every segment of its Newton polygon. For every segment $S$ of the Newton polygon $\mathscr{N}_{f}$ we denote by $|S|_{1}$ and $|S|_{2}$ the lengths of the projections of $S$ on the horizontal and vertical axes. We call $|S|_{1} /|S|_{2}$ the inclination of $S$.

A segment $S \in \mathscr{N}_{f}$ is exceptional if $1=|S|_{1}<|S|_{2}$ (resp. $1=|S|_{2}<|S|_{1}$ ) and $S$ has a vertex on the vertical (resp. horizontal) axis. Let $\mathcal{N}_{f}^{*}$ be the set of segments of $\mathscr{N}_{f}$ which are not exceptional. For every $S \in \mathscr{N}_{f}^{*}$ we consider the number $\varepsilon(S) \in\{-1,0,1\}$ defined as follows: $\varepsilon(S)=-1$ if $1<|S|_{1}<|S|_{2}$ (resp. $1<|S|_{2}<|S|_{1}$ ) and $S$ has a vertex on the vertical (resp. horizontal) axis, $\varepsilon(S)=$ 1 - (the number of vertices of $S$ lying on the axes) if $|S|_{1}=|S|_{2} ; \varepsilon(S)=0$ for all remaining cases. Let $\alpha / \alpha(S)+\beta / \beta(S)=1$ be the equation of the line containing $S$. Obviously $\alpha(S), \beta(S)>0$ are rational numbers and $\alpha(S) / \beta(S)=|S|_{1} /|S|_{2}$.

Now we can state our main result

THEOREM 1.1. Let $f=f(X, Y) \in C\{X, Y\}$ be a power series with an isolated singularity at $(0,0) \in C^{2}$. Then for every line $b X-a Y=0$ not tangent to the curve $f=0$ there is a factorization of the polar $\partial f=a(\partial f / \partial X)+b(\partial f / \partial Y)$ :

such that

$$
\partial f=v \prod_{S \in \mathcal{N}_{f}^{*}} g_{s} \text { in } \boldsymbol{C}\{X, Y\}
$$

(i) ord $g_{s}=\min \left(|S|_{1},|S|_{2}\right)+\varepsilon(S)$ for $S \in \mathscr{N}_{f}^{*}$,

(ii) if $h$ is an irreducible factor of $g_{s}$, then

$$
\frac{(f, h)_{0}}{\operatorname{ord} h} \geq \max (\alpha(S), \beta(S)),
$$

the equality holding if $f$ is nondegenerated on $S$.

(iii) if $\operatorname{ord} v>0$ then $\operatorname{ord} v=1$ and $(f, v)_{0}=\operatorname{ord} f$. 
The proof of the above theorem we give in Section 3 of this paper. Now, we state corollaries.

Corollary 1.2. Let $f=f(X, Y) \in C\{X, Y\}$ be a power series with an isolated singularity at $(0,0) \in C^{2}$ nondegenerate in Kouchnirenko's sense. Then

(i) the numbers $\max (\alpha(S), \beta(S))$ where $S \in \mathcal{N}_{f}^{*}$ are polar quotients of $f=0$,

(ii) if $q \neq \operatorname{ord} f$ is a polar quotient of $f$ then $q=\max (\alpha(S), \beta(S)$ ) for $a$ segment $S \in \mathscr{N}_{f}^{*}$.

The above corollary follows immediately from Theorem 1.1. One can complete (1.2) by noting that with the assumptions of (1.2) the number ord $f$ is a polar quotient of $f=0$ if and only if the line $\alpha+\beta=\operatorname{ord} f$ intersects $\operatorname{supp} f$ at a point not lying on the axes.

Using (1.2) and Teissier's formula for the Łojasiewicz exponent mentioned above we get the following corollary (see [Li], [F], [Len]):

COROLlary 1.3. If $f \in C\{X, Y\}$ has an isolated singularity and is nondegenerate in Kouchnirenko's sense, then

$$
\mathscr{L}_{0}(f)=\max \left\{\max (\alpha(S), \beta(S)): S \in \mathcal{N}_{f}^{*}\right\}-1
$$

if $\mathscr{N}_{f}^{*} \neq \emptyset$.

Note that $\mathscr{L}_{0}(f)=$ ord $f=2$ if $\mathscr{N}_{f}^{*}=\emptyset$.

COROLlaRY 1.4. Let $f=\sum_{\alpha w_{1}+\beta w_{2} \geq w} c_{\alpha \beta} X^{\alpha} Y^{\beta}$ where $f_{0}=$ $\sum_{\alpha w_{1}+\beta w_{2}=w} c_{\alpha \beta} X^{\alpha} Y^{\beta}$ has an isolated singularity of order $>2$. Then the number $\max \left(w / w_{1}, w / w_{2}\right)$ is a polar quotient of $f=0$ and there are no polar quotients of $f=0$ different from ord $f$ and $\max \left(w / w_{1}, w / w_{2}\right)$.

Proof. We have $\mathscr{N}_{f}=\{S\}$, where $S$ is a segment lying on the line $\alpha w_{1}+$ $\beta w_{2}=w$. It is easy to check that $S$ is not exceptional. Hence $\partial f=v g_{S}$ and $\max (\alpha(S), \beta(S))=\max \left(w / w_{1}, w / w_{2}\right)$. Then we use (1.2).

Examples. 1. If $f=X^{a}+Y^{b}$ where $a, b>1$ are integers, then there is the unique polar quotient and it is equal to $\max (a, b)$.

2. If $f=X^{a} Y+X Y^{b}$ where $a, b>1, a \neq b$ are integers, then there are two polar quotients: $\min (a, b)+1$ and $(a b-1) /(\min (a, b)-1)$.

3. If $f=Y^{8}+X Y^{5}+X^{3} Y^{2}+X^{6}$ then $\mathcal{N}_{f}=\{A, B, C\}$ where $A$ is exceptional, $B$ is of the first kind and $C$ is of the second kind. Here $\varepsilon(B)=0$, $\varepsilon(C)=-1$ and ord $g_{B}=2$, ord $g_{C}=1$. From $\partial f=v g_{B} g_{C}$ we get ord $v=1$. Hence there are three polar quotients: ord $f=5, \max (\alpha(B), \beta(B))=\beta(B)=$ $13 / 2, \max (\alpha(C), \beta(C))=\alpha(C)=6$. Moreover $\mathscr{L}_{0}(f)=(13 / 2)-1=11 / 2$. 


\section{Newton polygon of the polar curves}

Let $f \in C\{X, Y\}$ be a nonzero power series such that ord $f>1$. We assume that the distances $d_{1}, d_{2}$ from supp $f$ to the axes are less or equal to 1. Every series with an isolated singularity satisfies the above conditions.

Let $\partial=a(\partial / \partial X)+b(\partial / \partial Y)$ be such that $a b \neq 0$ and $\partial(\operatorname{in} f) \neq 0$ (note that $\partial($ in $f)=0$ if and only if in $f=$ const. $\left.(b X-a Y)^{\text {ord } f}\right)$. To avoid a trivial case we assume that $f$ is not generic with respect to $X, Y$ that is ord $f(X, 0) \neq$ ord $f$ or ord $f(0, Y) \neq$ ord $f$.

Our purpose is to compare the Newton polygons of the series $f$ and $\partial f$. We divide the nonexceptional segments of $\mathscr{N}_{f}$ into two classes. A segment $S \in \mathscr{N}_{f}$ is of the first kind if 1) $|S|_{1}<|S|_{2}$ and $S$ has no vertex on the vertical axis or 2) $|S|_{2}<|S|_{1}$ and $S$ has no vertex on the horizontal axis or 3) $|S|_{1}=|S|_{2}$ and $S$ has no vertices on the axes. Clearly, every segment of the first kind is not exceptional. A segment $S \in \mathscr{N}_{f}$ is of the second kind if it is neither of the first kind nor exceptional.

Let $S$ be a segment of the first kind with vertices $\left(\alpha_{1}, \beta_{1}\right)$ and $\left(\alpha_{2}, \beta_{2}\right)$ where $\alpha_{1}<\alpha_{2}$ (then $\beta_{1}>\beta_{2}$ ). We denote by $\partial S$ the segment with vertices $\left(\alpha_{1}-1, \beta_{1}\right)$ and $\left(\alpha_{2}-1, \beta_{2}\right)$ if $|S|_{1}<|S|_{2}$, the segment with vertices $\left(\alpha_{1}, \beta_{1}-1\right)$ and $\left(\alpha_{2}, \beta_{2}-1\right)$ if $|S|_{2}<|S|_{1}$ and the segment with vertices $\left(\alpha_{1}-1, \beta_{1}\right)$ and $\left(\alpha_{2}, \beta_{2}-1\right)$ if $|S|_{1}=|S|_{2}$. Clearly $S$ and $\partial S$ are parallel.

A vertex of the Newton diagram $\Delta(f)$ is principal if it is lying on the line $\alpha+\beta=\operatorname{ord} f$. If there is exactly one principal vertex $\left(\alpha_{0}, \beta_{0}\right)$ and it is not lying on the axes (that is $\alpha_{0}>0$ and $\beta_{0}>0$ ) then we denote by $V$ the segment with vertices $\left(\alpha_{0}-1, \beta_{0}\right)$ and $\left(\alpha_{0}, \beta_{0}-1\right)$. Clearly $|V|_{1}=|V|_{2}=1$. We set $\mathscr{V}=\{V\}$ if $V$ exists and $\mathscr{V}=\emptyset$, otherwise.

The first (last) segment of $\mathscr{N}_{f}$ is the segment nearest to the vertical (horizontal) axis. Every segment of the second kind is the first or is the last.

Let $\delta_{1}, \delta_{2} \geq 0$ be the distances from $\operatorname{supp}(\partial f)$ to the axes. We can state now the main result of this section.

THEOREM 2.1. With the assumptions and definitions introduced above, we have

(1) If $S \in \mathscr{N}_{f}$ is of the first kind then $\partial S \in \mathscr{N}(\partial f)$. If $\Delta(f)$ has exactly one principal vertex and it is not lying on the axes then $V \in \mathcal{N}(\partial f)$. The segments $\partial S$ $\left(S \in \mathscr{N}_{f}\right.$ of the first kind) and the segment $V$ form the sequence of succesive segments when ordered in such a way that the sequence of their inclinations is increased. If the first segment (the last) is of the first kind or it is exceptional, then $\delta_{1}=0\left(\delta_{2}=0\right)$.

(2) Suppose that the first segment $F \in \mathscr{N}_{f}$ is of inclination $\leq 1$ and $F$ has a vertex on the vertical axis. There is a set $\mathscr{T}_{1}$ of segments of $\mathscr{N}(\partial f)$ such that

(i) if $T \in \mathscr{T}_{1}$ then $|T|_{1} /|T|_{2} \leq|F|_{1} /|F|_{2}$,

(ii) $\delta_{1}+\sum_{T \in \mathscr{T}_{1}}|T|_{1}=|F|_{1}+\varepsilon(F)$, $(0, \beta(F))$.

(iii) a line supporting $\Delta(f)$ parallel to a segment $T \in \mathscr{T}_{1}$ passes through 
If the first segment is of inclination $>1$ or has no vertex on the vertical axis we put $\mathscr{T}_{1}=\emptyset$.

(3) Suppose that the last segment $L \in \mathscr{N}_{f}$ is of inclination $\geq 1$ and $L$ has a vertex on the horizontal axis. There is a set $\mathscr{T}_{2}$ of segments of $\mathscr{N}(\partial f)$ such that

(i') if $T \in \mathscr{T}_{2}$ then $|L|_{1} /|L|_{2} \leq|T|_{1} /|T|_{2}$,

(ii') $\delta_{2}+\sum_{T \in \mathscr{T}_{2}}|T|_{2}=|L|_{2}+\varepsilon(L)$, $(\alpha(L), 0)$.

(iii' $^{\prime}$ a line supporting $\Delta(f)$ parallel to a segment $T \in \mathscr{T}_{2}$ passes through

If the last segment is of inclination $<1$ or has no vertex on the horizontal axis we put $\mathscr{T}_{2}=\emptyset$.

(4) $\mathscr{N}(\partial f)=\left\{\partial S: S \in \mathscr{N}_{f}\right.$ is of the first kind $\} \cup \mathscr{V} \cup \mathscr{T}_{1} \cup \mathscr{T}_{2}$.

(5) Let $S \in \mathscr{N}_{f}$ and $T \in \mathscr{N}(\partial f)$ be parallel. Then

$$
\operatorname{in}(\partial f, T)=\left\{\begin{array}{l}
a \frac{\partial}{\partial X} \operatorname{in}(f, S) \text { if }|S|_{1}<|S|_{2}, \\
b \frac{\partial}{\partial Y} \operatorname{in}(f, S) \text { if }|S|_{2}<|S|_{1}, \\
\partial \operatorname{in}(f, S) \text { if }|S|_{1}=|S|_{1} .
\end{array}\right.
$$

In order to prove theorem 2.1 we need some preliminary notions. An application $v: C\{X, Y\} \backslash\{0\} \rightarrow \boldsymbol{N}$ is a weight of $\boldsymbol{C}\{X, Y\}$ if for every nonzero $f=\sum c_{\alpha \beta} X^{\alpha} Y^{\beta} \in C\{X, Y\}$ we have $v(f)=\inf \{\alpha v(X)+\beta v(Y):(\alpha, \beta) \in \operatorname{supp} f\}$, where $v(X)>0, v(Y)>0$ are integers. We put $v(0)=+\infty$ where the symbol $+\infty$ has usual properties. Moreover we put

$$
\operatorname{in}_{v} f=\sum_{\alpha v(X)+\beta v(Y)=v(f)} c_{\alpha \beta} X^{\alpha} Y^{\beta} .
$$

For every $S \in \mathcal{N}_{f}$ we consider the weight $v_{S}$ associated to $S$ by putting $v_{S}(X)=$ $|S|_{2}$ and $v_{S}(Y)=\left|S_{1}\right|$. Clearly $\alpha|S|_{2}+\beta|S|_{1}=v_{S}(f)$ is the equation of the line $l_{S}$ containing the segment $S$, therefore $v_{S}(f)=|S|_{2} \alpha(S)=|S|_{1} \beta(S)$. A line $l \subset$ $\boldsymbol{R}^{2}$ is a barrier of $\Delta(f)$ if it has equation $v_{1} \alpha+v_{2} \beta=w$ where $v_{1}, v_{2}, w>0$ are integers and satisfies two conditions: 1. if $(\alpha, \beta) \in \Delta(f)$, then $v_{1} \alpha+v_{2} \beta \geq w, 2$. $\Delta(f) \cap l \neq \emptyset$. We call $v_{2} / v_{1}$ the inclination of $l$. Clearly, the inclination of $l_{S}$ is equal to the inclination of $S$.

In the sequel we use the following simple facts:

1) $p \in \operatorname{supp} f$ is a vertex of $\Delta(f)$ if and only if there is a barrier $l$ of $\Delta(f)$ such that $\Delta(f) \cap l=\{p\}$.

2) vertices $p, p^{\prime}$ of $\Delta(f)$ are succesive if and only if the line passing through $p$ and $p^{\prime}$ is a barrier of $\Delta(f)$.

If $v$ is a weight of $C\{X, Y\}$, then the line $l_{v}: v(X) \alpha+v(Y) \beta=v(f)$ is a barrier of $\Delta(f)$ and $\Delta(f) \cap l_{v}=\operatorname{conv}\left(\operatorname{suppin}_{v} f\right)$. If $l$ is a barrier of $\Delta(f)$, then $l=l_{v}$ for a weight $v$. We omit the easy proof of the following lemma.

LEMMA 2.2. Let $\partial=a(\partial / \partial X)+b(\partial / \partial Y)$ with $(a, b) \neq(0,0)$. 
(i) If $a \neq 0$ and $v$ is a weight such that $v(Y)<v(X)$ and $\operatorname{in}_{v} f \neq$ const. $Y^{q}$ then $v(\partial f)=v(f)-v(X)$ and $\operatorname{in}_{v}(\partial f)=a(\partial / \partial X) \operatorname{in}_{v} f$,

(ii) If $b \neq 0$ and $v$ is a weight such that $v(X)<v(Y)$ and $\operatorname{in}_{v} f \neq$ const. $X^{p}$ then $v(\partial f)=v(f)-v(Y)$ and $\operatorname{in}_{v}(\partial f)=b(\partial / \partial Y) \operatorname{in}_{v} f$,

(iii) If in $f \neq$ const. $(b Y-a X)^{m}$ and $a b \neq 0$ then $\operatorname{ord}(\partial f)=\operatorname{ord} f-1$ and $\operatorname{in}(\partial f)=\partial(\operatorname{in} f)$.

For every $Z \subset \boldsymbol{R}^{2}$ we denote by $Z-(1,0)$ resp. $Z-(0,1)$ the image of $Z$ by the translation $(\alpha, \beta) \mapsto(\alpha-1, \beta)$ resp. $(\alpha, \beta) \mapsto(\alpha, \beta-1)$. Using the notion of barrier we can rewrite lemma 2.2 as follows.

LEMMA 2.3. (i) Let $\partial=a(\partial / \partial X)+b(\partial / \partial Y)$ with $a \neq 0$ and let $l$ be a barrier of $\Delta(f)$ of inclination $<1$ such that $l \cap \operatorname{supp} f$ contains a point not lying on the vertical axis. Then $l-(1,0)$ is a barrier of $\Delta(\partial f)$ and

$(l-(1,0)) \cap \operatorname{supp}(\partial f)=\left\{(\alpha-1, \beta) \in N^{2}: \alpha>0\right.$ and $\left.(\alpha, \beta) \in l \cap \operatorname{supp} f\right\}$.

(ii) Let $\partial=a(\partial / \partial X)+b(\partial / \partial Y)$ with $b \neq 0$ and let $l$ be a barrier of $\Delta(f)$ of inclination $>1$ such that $l \cap \operatorname{supp} f$ contains a point not lying on the horizontal axis. Then $l-(0,1)$ is a barrier of $\Delta(\partial f)$ and

$(l-(0,1)) \cap \operatorname{supp}(\partial f)=\left\{(\alpha, \beta-1) \in N^{2}: \beta>0\right.$ and $\left.(\alpha, \beta) \in l \cap \operatorname{supp} f\right\}$.

(iii) Let $\partial=a(\partial / \partial X)+b(\partial / \partial Y)$ be such that in $f \neq c o n s t .(b X-a Y)^{m}$. Then the line $k: \alpha+\beta=$ ord $f-1$ is a barrier of $\Delta(\partial f)$ and

$k \cap \operatorname{supp}(\partial f)$

$$
\begin{aligned}
& \subset\left\{(\alpha-1, \beta) \in N^{2}: \alpha>0,(\alpha, \beta) \in \operatorname{supp} f \text { and } \alpha+\beta=\operatorname{ord} f\right\} \\
& \cup\left\{(\alpha, \beta-1) \in N^{2}: \beta>0,(\alpha, \beta) \in \operatorname{supp} f \text { and } \alpha+\beta=\operatorname{ord} f\right\}
\end{aligned}
$$

Now we can prove Theorem 2.1 .

Proof of (1). Let $S \in \mathcal{N}_{f}$ be a segment of the first kind. Assume that $|S|_{1}<|S|_{2}$ (the cases $|S|_{2}<|S|_{1}$ and $|S|_{1}=|S|_{2}$ are treated similarly). Then $\partial S=S-(1,0)$. By Lemma 2.3 (i) the line $l_{S}-(1,0)$ is a barrier of $\operatorname{supp}(\partial f)$ and $l_{S}-(1,0)=l_{\partial S}$. Therefore $\partial S$ is a segment of $\mathscr{N}(\partial f)$.

To check the second part of (1) consider two succesive segments $S, S^{\prime}$ of $\mathscr{N}_{f}$. It suffices to observe that

1. If $S, S^{\prime}$ are both of inclination $\leq 1$ or $\geq 1$, then $\partial S, \partial S^{\prime}$ are succesive segments of $\mathscr{N}(\partial f)$,

2. If $|S|_{1} /|S|_{2}<1<\left|S^{\prime}\right|_{1} /\left|S^{\prime}\right|_{2}$, then the common vertex of $S$ and $S^{\prime}$ is principal and $\partial S, V, \partial S^{\prime}$ are succesive segments of $\mathscr{N}(\partial f)$.

3. If $|S|_{1}<|S|_{2}$, the lower vertex of $S$ does not lie on the horizontal axis and is principal (resp. $|S|_{2}<|S|_{1}$ and the upper vertex of $S$ does not lie on the vertical axis), then $\partial S, V$ (resp. $V, \partial S$ ) are successive segments of $\mathscr{N}(\partial f)$. To end the proof of (1) let us observe that if $F$ is of the first kind then the segment $\partial F$ has a 
vertex on the vertical axis. If $F$ is exceptional with the lower vertex $(1, \beta)$, then the point $(0, \beta)$ is a vertex of $\mathscr{N}(\partial f)$. Therefore $\delta_{1}=0$. Analogously we treat the case of the last segment.

Proof of (2). Let $F$ be the first segment of $\mathscr{N}_{f}$. Suppose that $F$ is not exceptional, $|F|_{1} \leq|F|_{2}$ and $F$ has a vertex on the vertical axis. Let $\left(\alpha_{0}, \beta_{0}\right)=$ $\left(0, \beta_{0}\right)=(0, \beta(F))$ and $\left(\alpha_{1}, \beta_{1}\right)=\left(|F|_{1}, \beta_{1}\right)$ be vertices of $F$. Let $\left(\alpha_{1}^{\prime}, \beta_{1}^{\prime}\right)=$ $\left(\alpha_{1}-1, \beta_{1}\right)$ if $|F|_{1}<|F|_{2}$ and $\left(\alpha_{1}^{\prime}, \beta_{1}^{\prime}\right)=\left(\alpha_{1}, \beta_{1}-1\right)$ if $|F|_{1}=|F|_{2}$. We claim that $\left(\alpha_{1}^{\prime}, \beta_{1}^{\prime}\right)$ is a vertex od $\Delta(\partial f)$.

Indeed, there is a barrier $l$ of $\Delta(f)$ such that $l \cap \operatorname{supp} f=\left\{\left(\alpha_{1}, \beta_{1}\right)\right\}$ and the inclination of $l$ is $<1$ if $|F|_{1}<|F|_{2}$ and $>1$ if $|F|_{1}=|F|_{2}$. Therefore $(l-(1,0)) \cap \operatorname{supp}(\partial f)=\left\{\left(\alpha_{1}^{\prime}, \beta_{1}^{\prime}\right)\right\}$ if $\left.|F|_{1}<|F|_{2},(l-(0,1)) \cap \operatorname{supp}(\partial f)=\left\{\alpha_{1}^{\prime}, \beta_{1}^{\prime}\right)\right\}$ if $|F|_{1}=|F|_{2}$ and $\left(\alpha_{1}^{\prime}, \beta_{1}^{\prime}\right)$ is a vertex of $\Delta(\partial f)$ by Lemma 2.3. Let $\mathscr{T}_{1}$ be the set of all segments $T \in \mathscr{N}(\partial f)$ lying above the vertex $\left(\alpha_{1}^{\prime}, \beta_{1}^{\prime}\right)$. We will check properties (i), (ii), (iii) when $|F|_{1}<|F|_{2}$ (the proofs when $|F|_{1}=|F|_{2}$ are similar). By Lemma 2.3 the line $l_{F}-(1,0)$ is a barrier of $\Delta(\partial f)$ passing through the vertex $\left(\alpha_{1}-1, \beta_{1}\right)$. Let $T \in \mathscr{T}_{1}$ and consider the barrier $l_{F}$ of $\Delta(\partial f)$. Clearly $|T|_{1} /|T|_{2}=$ inclination of $l_{T} \leq$ inclination of $l_{F}-(1,0)=|F|_{1} /|F|_{2}$, that is (i) holds.

Property (ii) follows from the calculation:

$$
\delta_{1}+\sum_{T \in \mathscr{T}_{1}}|T|_{1}=\alpha_{1}-1=|F|_{1}-1=|F|_{1}+\varepsilon(F) .
$$

To check (iii) take a barrier of $\Delta(f)$ parallel to a segment of $T \in \mathscr{T}_{1}$. It is of inclination $|T|_{1} /|T|_{2} \leq|F|_{1} /|F|_{2}$. Therefore it passes through $\left(0, \beta_{0}\right)=(0, \beta(F))$.

Proof of (3). The proof of (3) is analogous to that of (2).

Proof of (4). (4) follows easily from (1), (2) and (3).

Proof of (5). Consider two parallel segments $S \in \mathscr{N}(f)$ and $T \in \mathscr{N}(\partial f)$. Let $v_{S}$ be the weight defined by putting $v_{S}(X)=|S|_{2}$ and $v_{S}(Y)=|S|_{1}$. Assume $|S|_{1}<|S|_{2}$. Clearly $\operatorname{in}_{v_{S}} f=\operatorname{in}(f, S) \neq \mathrm{a}$ monomial and by Lemma 2.2 (i): $\operatorname{in}_{v_{S}}(\partial f)=a(\partial / \partial X) \operatorname{in}(f, S)$. On the other hand $\operatorname{in}_{v_{S}}(\partial f)=\operatorname{in}_{v_{T}}(\partial f)$ for $S$ and $T$ are parallel and we get

$$
\operatorname{in}(\partial f, T)=\operatorname{in}_{v_{T}}(\partial f)=\operatorname{in}_{v_{S}}(\partial f)=a \frac{\partial}{\partial X} \operatorname{in}(f, S) .
$$

If $|S|_{2}<|S|_{1}$ or $|S|_{2}=|S|_{1}$, then the proof is similar.

\section{Proof of the main result}

We need some preliminary facts. The following is well-known (see [Te2]).

LEMMA 3.1. Let $f=f(X, Y)$ be a nonzero power series without constant term. Then there is a factorization 


$$
f=u X^{d_{1}} Y^{d_{2}} \prod_{S \in \mathscr{N}_{f}} f_{S}
$$

in $C\{X, Y\}$ where $u$ is a unit such that

(i) $\mathscr{N}\left(f_{S}\right)=\left\{S^{\prime}\right\}$ where $S^{\prime}$ is the segment with vertices $\left(|S|_{1}, 0\right)$ and $\left(0,|S|_{2}\right)$,

(ii) in $(f, S)=($ a monomial $)$ in $\left(f_{S}, S^{\prime}\right)$ in $C\{X, Y\}$.

Recall that a pair of power series $f, g$ without constant term is nondegenerate if for every pair of parallel segments $S \in \mathcal{N}_{f}$ and $T \in \mathscr{N}_{g}$ the system of equations in $(f, S)=\operatorname{in}(g, T)=0$ has no solution in $C^{*} \times C^{*}$. Let

$$
\frac{\alpha}{\alpha\left(T, \mathscr{N}_{f}\right)}+\frac{\beta}{\beta\left(T, \mathscr{N}_{f}\right)}=1
$$

be the equation of the barrier of $\Delta(f)$ parallel to the segment $T$ of (possibly) another Newton polygon. Clearly, if $S \in \mathscr{N}_{f}$, then $\alpha\left(S, \mathscr{N}_{f}\right)=\alpha(S), \beta\left(S, \mathscr{N}_{f}\right)=$ $\beta(S)$.

LEMMA 3.2. Let $f \in C\{X, Y\}$ be a nonzero power series without constant term and let $h \in C\{X, Y\}$ be a power series such that $\mathscr{N}_{h}=\{T\}$ where $T$ is a segment with vertices on the axes. Then

$$
\frac{(f, h)_{0}}{\operatorname{ord} h} \geq \max \left(\alpha\left(T, \mathscr{N}_{f}\right), \beta\left(T, \mathscr{N}_{f}\right)\right)
$$

the equality holding if the pair $f, h$ is nondegenerate.

Recall the following (see, for example [Pł2]):

PROPERTY. Let $f, g$ be nonzero power series without constant term. Then for every weight $v$ :

$$
(f, g)_{0} \geq \frac{v(f) v(g)}{v(X) v(Y)}
$$

the equality holding if the system of equations $\operatorname{in}_{v} f=\operatorname{in}_{v} g=0$ has no solution $\neq(0,0)$.

To check Lemma 3.2 we apply the above property of $f, h$ and to the weight $v_{T}$ given by $v_{T}(X)=|T|_{2}$ and $v_{T}(Y)=|T|_{1}$. It is easy to see that $v_{T}(h)=$ $|T|_{1}|T|_{2}, \quad v_{T}(f)=|T|_{2} \alpha\left(T, \mathscr{N}_{f}\right)=|T|_{1} \beta\left(T, \mathscr{N}_{f}\right) \quad$ and $\quad$ ord $h=\min \left(|T|_{1},|T|_{2}\right)$. Therefore

$$
\begin{aligned}
\frac{(f, h)_{0}}{\operatorname{ord} h} & \geq \frac{v_{T}(f)}{\min \left(|T|_{1},|T|_{2}\right)}=\max \left(\frac{v_{T}(f)}{|T|_{1}}, \frac{v_{T}(f)}{|T|_{2}}\right) \\
& =\max \left(\alpha\left(T, \mathscr{N}_{f}\right), \beta\left(T, \mathscr{N}_{f}\right)\right),
\end{aligned}
$$

the equality holding if the pair $f, h$ is nondegenerate. 
LEMMA 3.3. Every polar quotient of the curve $f=0$ is greater than or equal to ord $f$. If the curve $f=0$ has ord $f$ tangents paiwise different, then there is only one polar quotient of $f=0$ and it is equal to ord $f$.

Proof. Suppose that the line $b X-a Y=0$ is not tangent to $f=0$. The first part follows from the inequality $(f, h)_{0} \geq(\operatorname{ord} f)(\operatorname{ord} h)$. It is easy to check that if $f$ has ord $f$ tangents, then the curves $f=0$ and $\partial f=a \partial f / \partial X+b \partial f / \partial Y=$ 0 have no common tangent. Therefore for every irreducible factor $h$ of $\partial f$ we get

$$
\frac{(f, h)_{0}}{\operatorname{ord} h}=\operatorname{ord} f
$$

Proof of Theorem 1.1. Let $f=f(X, Y) \in C\{X, Y\}$ be a power series with an isolated singularity at $(0,0) \in C^{2}$. If $\mathscr{N}_{f}=\{S\},|S|_{1}=|S|_{2}$ or $\mathscr{N}_{f}^{*}=\emptyset$ then the theorem follows easily from Lemma 3.3. Therefore we assume in the sequel that $\mathscr{N}_{f}$ contains a segment of inclination $\neq 1$.

Let $\partial=a \partial / \partial X+b \partial / \partial Y$ with $a b$ in $f(a, b) \neq 0$. Then $\partial($ in $f) \neq 0$. By Lemma 3.1 we may write

$$
\partial f=u X^{\delta_{1}} Y^{\delta_{2}} \prod_{T \in \mathscr{N}(\partial f)}(\partial f)_{T} .
$$

For every nonexceptional segment $S \in \mathcal{N}_{f}$ we define the series $g_{S}$ as follows. If $S$ is a segment of the first kind, then $g_{S}=(\partial f)_{\partial S}$. If $F$ is the first segment of inclination $\leq 1$ and with a vertex on the horizontal axis, then we put

$$
g_{F}=X^{\delta_{1}} \prod_{T \in \mathscr{T}_{1}}(\partial f)_{T}
$$

Similarly, if $L$ is the last segment of inclination $\geq 1$ and it is of the second kind, then

$$
g_{L}=Y^{\delta_{2}} \prod_{T \in \mathscr{T}_{2}}(\partial f)_{T}
$$

Moreover, we put $v=u(\partial f)_{V}$ if $\mathscr{V}=\{V\}$ and $v=u$ if $\mathscr{V}=\emptyset . \quad$ By Theorem 2.1 and (1) we get

$$
\partial f=v \prod_{S \in \mathcal{N}_{f}^{*}} g_{S}
$$

To check Theorem 1.1 we have to verify three cases.

CASE 1. $S$ is a segment of the first kind. Recall that for every segment of the first kind, $\varepsilon(S)=0$ if $|S|_{1} \neq|S|_{2}$ and $\varepsilon(S)=1$ if $|S|_{1}=|S|_{2}$. Therefore

$$
\operatorname{ord} g_{S}=\operatorname{ord}(\partial f)_{\partial S}=\min \left(|\partial S|_{1},|\partial S|_{2}\right)=\min \left(|S|_{1},|S|_{2}\right)+\varepsilon(S)
$$

This proves part (i) of the theorem. 
Let $h$ be an irreducible factor of $g_{S}=(\partial f)_{\partial S}$. Then $\mathscr{N}_{h}=\{T\}, T$ is parallel to $\partial S$ and consequently to $S$. By Lemma 3.2 we get

$$
\begin{aligned}
\frac{(f, h)_{0}}{\operatorname{ord} h} & \geq \max \left(\alpha\left(T, \mathcal{N}_{f}\right), \beta\left(T, \mathcal{N}_{f}\right)\right)=\max \left(\alpha\left(S, \mathcal{N}_{f}\right), \beta\left(S, \mathcal{N}_{f}\right)\right) \\
& =\max (\alpha(S), \beta(S)) .
\end{aligned}
$$

If $f$ is nondegenerate on $S$, then by part (5) of Theorem 2.1 the pair $f, h$ is nondegenerate and we get

$$
\frac{(f, h)_{0}}{\operatorname{ord} h}=\max (\alpha(S), \beta(S))
$$

CASE 2. The first segment $F,|F|_{1} \leq|F|_{2}$ of $\mathcal{N}_{f}$ is of the second kind. We get by the second part of Theorem 2.1:

$$
\begin{aligned}
\operatorname{ord} g_{F} & =\operatorname{ord}\left(X_{1}^{\delta} \prod_{T \in \mathscr{T}_{1}}(\partial f)_{T}\right)=\delta_{1}+\sum_{T \in \mathscr{T}_{1}} \operatorname{ord}(\partial f)_{T} \\
& =\delta_{1}+\sum_{T \in \mathscr{T}_{1}}|T|_{1}=|F|_{1}+\varepsilon(F)=\min \left(|F|_{1},|F|_{2}\right)+\varepsilon(F) .
\end{aligned}
$$

Let $h$ be an irreducible factor of $g_{F}$. If $h$ divides $(\partial f)_{T}$, then $\mathscr{N}_{h}=\left\{T^{\prime}\right\}$ where $T^{\prime}$ is parallel to $T$. Thus $\alpha\left(T^{\prime}, \mathscr{N}_{f}\right)=\alpha\left(T, \mathcal{N}_{f}\right), \beta\left(T^{\prime}, \mathcal{N}_{f}\right)=\beta\left(T, \mathscr{N}_{f}\right)$ and we get

$$
\frac{(f, h)_{0}}{\operatorname{ord} h} \geq \max \left(\alpha\left(T^{\prime}, \mathscr{N}_{f}\right), \beta\left(T^{\prime}, \mathscr{N}_{f}\right)\right)=\beta\left(T, \mathscr{N}_{f}\right)=\beta(F)
$$

by the second part of Theorem 2.1. The equality for $f$ nondegenerate on $S$ we check like in Case 1 . If $h=X$, then

$$
\frac{(f, h)_{0}}{\operatorname{ord} h}=\operatorname{ord} f(0, Y)=\beta(F)
$$

since the first segment $F$ has a vertex on the vertical axis.

CASE 3. The last segment $L$ of $\mathscr{N}_{f},|L|_{2} \leq|L|_{1}$ is of the second kind. The proof is analogous to the proof above.

To finish the proof it suffices to remark that if $\operatorname{ord} v>0$, then $\operatorname{ord} v=$ $\max \left(|V|_{1},|V|_{2}\right)=1$ and

$$
(f, v)_{0}=\max \left(\alpha\left(V, \mathscr{N}_{f}\right), \beta\left(V, \mathscr{N}_{f}\right)\right)=\text { ord } f
$$

since $|V|_{1}=|V|_{2}$ and the pair $f, v$ is nondegenerate.

We have proved our theorem for polars $\partial f=a \partial / \partial X+b \partial / \partial Y$ in the direction $(a: b)$ such that $a b$ in $f(a, b) \neq 0$. If $a=0$ or $b=0$ and in $f(a, b) \neq 0$ then the theorem is still true but the proof needs some modification: instead of using Theorem 2.1 we use the description of the polygons $\mathscr{N}(\partial f / \partial X), \mathscr{N}(\partial f / \partial Y)$ given in [Len] (Theorems 5.3 and 5.5). 
Remark. One can also prove factorization theorems similar to our main result for special polars $\partial f / \partial X$ and $\partial f / \partial Y$.

\section{REFERENCES}

[Ab.A] S. S. Abhyankar AND A. Assi, Jacobian of meromorphic curves, Prépublications de l'Université d'Angers, 1998.

[BK] E. BRIESKORN AND H. KNÖRER, Ebene Algebra1sche Kurven, Birkhäuser, 1981.

[C] E. CASAS, On the singularities of polar curves, Manuscripta Math., 43 (1983), 167-180.

[D] F Delgado De La Mata, A factorization theorem for the polar of a curve with two branches, Compositio Math., 92 (1994), 327-375.

[Fu] T. Fukui, Łojasiewicz type inequalities and Newton diagrams, Proc. Amer. Math. Soc., 112 (1991), 1169-1183.

[G] E. G. Barroso, Un théorème de décomposition pour les polaires génériques d'une courbe plane, C. R. Acad. Scı. Parıs Sér. I Math., 326 (1998), 59-62.

[KL] T. C. KUO AND Y C. LU, On analytic function germ of two complex variables, Topology, 16 (1997), 299-310.

[Kou] A. G. Kouchnirenko, Polyèdres de Newton et nombres de Milnor, Invent. Math., 32 (1976), 1-31.

[Len] A. LENARCIK, On the Lojasiewicz exponent of the gradient of a holomorphic function, Singularities Symposium-Lojasıewicz 70 (B. Jakubczyk ed.), Banach Center Publications, 44, Warszawa, 1998, 149-166.

[Li] B. Lichtin, Estımation of Lojasiewicz exponent and Newton polygons, Invent. Math., 64 (1981), 417-429.

[LMW1] Lê Dung Trang, F Michel and C. Weber, Sur le comportement des polares associées aux germes de courbes planes, Compositio Math., 72 (1989), 88-113.

[LMW2] $\longrightarrow$ Courbes polaires et topologie des courbes planes, Ann. Scı. École Norm. Sup. (4), 24 (1991), 141-169.

[M] M. Merle, Invariants polaires des courbes planes, Invent. Math., 41 (1977), 103-111.

[Pł1] A. Płoski, Newton polygons and the Łojasiewicz exponent of a holomorphic mapping of $C^{2}$, Ann. Polon. Math., 51 (1990), 275-281.

[Pł2] — Sur l'exposant d'une application analytique II, Bull. Acad. Pol. Math., 33 (1985), 123-127

[Tel] B. TeISSIER, Variétés polaires, Invent. Math., 40 (1977), 267-292.

[Te2] _ Introduction to curve singularities, Singularity Theory (D. T. Lê, K. Saito and B. Teissier eds.), World Scientific, 1995.

Department of Mathematics

TECHNICAL UNIVERSITY

AL. 1000 L PP 7

25-314 KIELCE, POLAND

E-mail: \{lenarcik,ploski\}@sabat.tu.kıelce.pl 